\title{
Palladium Catalyzed Arylation of Trimethylsilyl Enolates of Esters and Imides. High Functional Group Tolerance and Stereoselective Synthesis of $\square$-Aryl Carboxylic Acid Derivatives
}

Xiaoxiang Liu and John F. Hartwig*

Department of Chemistry, Yale University P.O. Box 208107, New Haven, CT 06520-8107

\section{Supporting Information}

\section{Full Experimental Section}

General Methods. Reactions were conducted using standard drybox techniques.

${ }^{1} \mathrm{H}$ and ${ }^{13} \mathrm{C}$ NMR spectra were recorded in $\mathrm{CDCl}_{3}$ on a $400 \mathrm{MHz}$ spectrometer with tetramethylsilane or residual protiated solvent used as a reference and coupling constants reported in Hertz $(\mathrm{Hz})$. Chromatographic purifications were performed by flash chromatography using silica gel (200-400 mesh) or using an automated chromatography system. Yields for final products in all tables refer to isolated yields and are the average of two runs. Products that had been reported previously were isolated in greater than $95 \%$ purity, as determined by ${ }^{1} \mathrm{H}$ NMR and capillary gas chromatography (GC). All ${ }^{13} \mathrm{C}$ NMR spectra were proton decoupled. GC analyses were obtained with a DB-1301 narrow bore column for high temperature ramp applications (max. $120{ }^{\circ} \mathrm{C} / \mathrm{min}$ ). Methyl trimethylsilyl ketene acetal, $\mathrm{ZnF}_{2}$ and $\mathrm{Zn}\left(\mathrm{O}^{t} \mathrm{Bu}\right)_{2}$ were purchased from commercial supplies. The silyl ketene acetal of $t$-butyl propionate ${ }^{1}$ and the trimethylsilyl ether derivative of the Evans auxiliary ${ }^{2}$ were prepared according to literature procedures. The racemic version\$Ley, 2001 \#75\& of the available non-racemic Ley auxiliary\$Diez, 2001 \#49\& was used to determine diastereoselectivities and was prepared by literature procedures.

\section{Representive Procedure for the Arylation of Silyl Ketene Acetals.}

Methyl phenylisobutyrate, (Table 2, entry 1). ${ }^{3}$ To a screw-capped vial containing $\mathrm{P}^{t} \mathrm{Bu}_{3}(40 \square \mathrm{L}$ of a $0.500 \mathrm{M}$ solution in toluene, $0.020 \mathrm{mmol}), \mathrm{Pd}(\mathrm{dba})_{2}(5.8 \mathrm{mg}, 0.010$ 
$\mathrm{mmol}), \mathrm{ZnF}_{2}(52.0 \mathrm{mg}, 0.500 \mathrm{mmol})$ and phenyl bromide $(157.0 \mathrm{mg}, 1.000 \mathrm{mmol})$ was added tert-butyl trimethylsilyl methyl ketene acetal $(301.0 \mathrm{mg}, 1.49 \mathrm{mmol}$ ), followed by DMF $(4.0 \mathrm{~mL})$. The vial was sealed with a cap containing a PTFE septum and removed from the dry box. The heterogeneous reaction mixture was stirred at $80{ }^{\circ} \mathrm{C}$ for $12 \mathrm{~h}$. The crude reaction was then allowed to cool to room temperature and was diluted with $\mathrm{Et}_{2} \mathrm{O}(50 \mathrm{~mL})$. The resulting solution was washed with $\mathrm{H}_{2} \mathrm{O}(5 \mathrm{X} 20 \mathrm{~mL})$. The organic phase was dried over $\mathrm{Na}_{2} \mathrm{SO}_{4}$, filtered, and concentrated at reduced pressure. The residue was then purified by chromatography on silica gel eluting with a 1-2\% gradient of EtOAc in hexane to provide the title compound (162 mg ) in 91\% yield. ${ }^{1} \mathrm{H}$ NMR $\left(\mathrm{CDCl}_{3}\right): \square 7.27-7.38(5 \mathrm{H}, \mathrm{m}), 3.69(3 \mathrm{H}, \mathrm{s}), 1.62(6 \mathrm{H}, \mathrm{s}) .{ }^{13} \mathrm{C} \mathrm{NMR}\left(\mathrm{CDCl}_{3}\right): \square 177.72$, 145.08, 128.85, 127.15, 126.02, 52.64, 46.94, 26.98.

Methyl (4-nitrophenyl)isobutyrate, (Table 2, entries 2 and 12). ${ }^{4}$

${ }^{1} \mathrm{H}$ NMR $\left(\mathrm{CDCl}_{3}\right): \square 8.20(2 \mathrm{H}, \mathrm{d}, J=9.0 \mathrm{~Hz}), 7.52(2 \mathrm{H}, \mathrm{d}, J=9.0 \mathrm{~Hz}), 3.70(3 \mathrm{H}, \mathrm{s}), 1.64$ $(6 \mathrm{H}, \mathrm{s}) .{ }^{13} \mathrm{C} \mathrm{NMR}\left(\mathrm{CDCl}_{3}\right): \square 176.44,152.35,147.08,127.25,124.01,52.97,47.34$, 26.79 .

tert-Butyl (4-nitrophenyl)propionate, (Table 2, entry 3).

${ }^{1} \mathrm{H}$ NMR $\left(\mathrm{CDCl}_{3}\right): \square 8.19(2 \mathrm{H}, \mathrm{d}, J=8.7 \mathrm{~Hz}), 7.47(2 \mathrm{H}, \mathrm{d}, J=8.7 \mathrm{~Hz}), 3.74(1 \mathrm{H}, \mathrm{q}, J=$ $7.2 \mathrm{~Hz}), 1.50(3 \mathrm{H}, \mathrm{d}, J=7.2 \mathrm{~Hz}), 1.40(9 \mathrm{H}, \mathrm{s}) .{ }^{13} \mathrm{C} \mathrm{NMR}\left(\mathrm{CDCl}_{3}\right): \square 172.81,148.90$, 147.39, 128.84, 124.13, 81.75, 46.83, 28.26, 18.71. Anal. Calcd for $\mathrm{C}_{13} \mathrm{H}_{17} \mathrm{NO}_{4}$ : C, 62.14; H, 6.82; N, 5.57. Found: C, 62.32; H, 6.98; N, 5.74.

Methyl (4-methoxycarbonylphenyl)isobutyrate, (Table 2, entry 4, 13). ${ }^{5}$

${ }^{1} \mathrm{H}$ NMR $\left(\mathrm{CDCl}_{3}\right): \square 8.01(2 \mathrm{H}, \mathrm{d}, J=8.4 \mathrm{~Hz}), 7.42(2 \mathrm{H}, \mathrm{d}, J=8.4 \mathrm{~Hz}), 3.93(3 \mathrm{H}, \mathrm{s}), 3.68$

$(3 \mathrm{H}, \mathrm{s}), 1.62(6 \mathrm{H}, \mathrm{s}) \cdot{ }^{13} \mathrm{C} \mathrm{NMR}\left(\mathrm{CDCl}_{3}\right): \square 177.02,167.18,150.16,130.12,128.99$, $126.16,52.74,52.47,47.19,26.79$.

tert-Butyl (4-methoxycarbonylphenyl)propionate, (Table 2, entry 5).

${ }^{1} \mathrm{H}$ NMR $\left(\mathrm{CDCl}_{3}\right): \square 8.00(2 \mathrm{H}, \mathrm{d}, J=8.3 \mathrm{~Hz}), 7.37(2 \mathrm{H}, \mathrm{d}, J=8.3 \mathrm{~Hz}), 3.92(3 \mathrm{H}, \mathrm{s}), 3.68$ $(1 \mathrm{H}, \mathrm{q}, J=7.1 \mathrm{~Hz}), 1.47(3 \mathrm{H}, \mathrm{d}, J=7.1 \mathrm{~Hz}), 1.39(9 \mathrm{H}, \mathrm{s}) .{ }^{13} \mathrm{C} \mathrm{NMR}\left(\mathrm{CDCl}_{3}\right): \square 173.48$, 
167.35, 146.76, 130.21, 129.20, 127.91, 81.25, 52.44, 46.94, 28.28, 18.67. Anal. Calcd for $\mathrm{C}_{15} \mathrm{H}_{20} \mathrm{O}_{4}$ : C, 68.16; H, 7.63. Found: $\mathrm{C}, 68.07 ; \mathrm{H}, 7.88$.

tert-Butyl (2-cyanophenyl)propionate, (Table 2, entry 6).

${ }^{1} \mathrm{H}$ NMR $\left(\mathrm{CDCl}_{3}\right): \square 7.66(1 \mathrm{H}, \mathrm{dd}, J=7.8$ and $1.0 \mathrm{~Hz}), 7.58(1 \mathrm{H}, \mathrm{dt}, J=7.6$ and $1.2 \mathrm{~Hz})$, $7.47(1 \mathrm{H}, \mathrm{d}, J=7.9 \mathrm{~Hz}), 7.36(1 \mathrm{H}, \mathrm{dt}, J=7.6$ and $1.0 \mathrm{~Hz}), 4.11(1 \mathrm{H}, \mathrm{q}, J=7.2 \mathrm{~Hz}), 1.52$ $(3 \mathrm{H}, \mathrm{d}, J=7.2 \mathrm{~Hz}), 1.42(9 \mathrm{H}, \mathrm{s}) .{ }^{13} \mathrm{C} \mathrm{NMR}\left(\mathrm{CDCl}_{3}\right): \square 172.52,145.33,133.99,133.32$, 127.78, 127.74, 118.16, 113.09, 81.86, 44.84, 28.27, 18.45. Anal. Calcd for $\mathrm{C}_{14} \mathrm{H}_{17} \mathrm{NO}_{2}$ : C, 72.70; H, 7.41; N, 6.06. Found: C, 72.43; H, 7.21; N, 6.24.

tert-Butyl (3-cyanophenyl)propionate, (Table 2, entry 7).

${ }^{1} \mathrm{H}$ NMR $\left(\mathrm{CDCl}_{3}\right): \square 7.60(1 \mathrm{H}, \mathrm{s}), 7.55-7.57(2 \mathrm{H}, \mathrm{m}), 7.44(1 \mathrm{H}, \mathrm{t}, J=7.8 \mathrm{~Hz}), 3.66(1 \mathrm{H}$, q, $J=7.2 \mathrm{~Hz}), 1.48(3 \mathrm{H}, \mathrm{d}, J=7.2 \mathrm{~Hz}), 1.41(9 \mathrm{H}, \mathrm{s}) .{ }^{13} \mathrm{C} \mathrm{NMR}\left(\mathrm{CDCl}_{3}\right): \square 173.09$, $142.88,132.49,131.65,131.08,129.69,119.19,112.98,81.65,46.49,28.03$, 18.71. Anal. Calcd for $\mathrm{C}_{14} \mathrm{H}_{17} \mathrm{NO}_{2}$ : C, 72.70; H, 7.41; N, 6.06. Found: C, 72.59; H, 7.46; N, 6.21.

\section{Methyl (4-benzoylphenyl)isobutyrate, (Table 2, entry 8). ${ }^{3}$}

${ }^{1} \mathrm{H}$ NMR $\left(\mathrm{CDCl}_{3}\right): \square 7.79-7.84(4 \mathrm{H}, \mathrm{m}), 7.61(1 \mathrm{H}, \mathrm{t}, J=7.4 \mathrm{~Hz}), 7.45-7.52(4 \mathrm{H}, \mathrm{m}), 3.70$ $(3 \mathrm{H}, \mathrm{s}), 1.65(6 \mathrm{H}, \mathrm{s}) .{ }^{13} \mathrm{C} \mathrm{NMR}\left(\mathrm{CDCl}_{3}\right): \square 196.67,177.10,149.74,137.98,136.38$, $132.84,130.73,130.44,128.70,126.12,52.84,47.25,26.86$.

\section{Methyl (4-acetylphenyl)isobutyrate, (Table 2, entry 9).}

${ }^{1} \mathrm{H}$ NMR $\left(\mathrm{CDCl}_{3}\right): \square 7.93(2 \mathrm{H}, \mathrm{d}, J=8.5 \mathrm{~Hz}), 7.43(2 \mathrm{H}, \mathrm{d}, J=8.5 \mathrm{~Hz}), 3.67(3 \mathrm{H}, \mathrm{s}), 2.60$ $(3 \mathrm{H}, \mathrm{s}), 1.61(6 \mathrm{H}, \mathrm{s}) .{ }^{13} \mathrm{C} \mathrm{NMR}\left(\mathrm{CDCl}_{3}\right): \square 198.01,176.98,150.39,136.04,128.92$, 126.37, 52.77, 47.24, 26.98, 26.79. Anal. Calcd for $\mathrm{C}_{13} \mathrm{H}_{16} \mathrm{O}_{3}$ : C, 70.89; H, 7.32. Found: C, 70.66; H, 7.60.

tert-Butyl (4-acetylphenyl)propionate, (Table 2, entry 10).

${ }^{1} \mathrm{H}$ NMR $\left(\mathrm{CDCl}_{3}\right): \square 7.93(2 \mathrm{H}, \mathrm{d}, J=8.3 \mathrm{~Hz}), 7.40(2 \mathrm{H}, \mathrm{d}, J=8.3 \mathrm{~Hz}), 3.68(1 \mathrm{H}, \mathrm{q}, J=$ 7.1Hz), $2.60(3 \mathrm{H}, \mathrm{s}), 1.48(3 \mathrm{H}, \mathrm{d}, J=7.2 \mathrm{~Hz}), 1.40(9 \mathrm{H}, \mathrm{s}) .{ }^{13} \mathrm{C} \mathrm{NMR}\left(\mathrm{CDCl}_{3}\right): \square 198.16$, 
173.42, 146.98, 136.27, 129.01, 128.10, 81.32, 46.93, 28.30, 26.99, 18.70. Anal. Calcd for $\mathrm{C}_{15} \mathrm{H}_{20} \mathrm{O}_{3}$ : C, 72.55; H, 8.12. Found: $\mathrm{C}, 72.31 ; \mathrm{H}, 8.15$.

Methyl (4-methoxyphenyl)isobutyrate, (Table 2, entry 11). ${ }^{\mathbf{3}}$

${ }^{1} \mathrm{H} \mathrm{NMR}\left(\mathrm{CDCl}_{3}\right): \square 7.29(2 \mathrm{H}, \mathrm{d}, J=8.8 \mathrm{~Hz}), 6.88(2 \mathrm{H}, \mathrm{d}, J=8.9 \mathrm{~Hz}), 3.82(3 \mathrm{H}, \mathrm{s}), 3.67$ $(3 \mathrm{H}, \mathrm{s}), 1.59(6 \mathrm{H}, \mathrm{s}) .{ }^{13} \mathrm{C}$ NMR $\left(\mathrm{CDCl}_{3}\right): \square 177.90,158.63,137.16,127.15,114.11$, $55.65,52.63,46.16,27.05$.

Benzyloxy-phenyl-acetic acid tert-butyl ester (table 4, entry 1): ${ }^{1} \mathrm{H}$ NMR $\left(\mathrm{CDCl}_{3}\right): \square$ 7.17-7.38 (10H, m), $4.73(1 \mathrm{H}, \mathrm{s}), 4.54(1 \mathrm{H}, \mathrm{d}, \mathrm{J}=9.5 \mathrm{~Hz}), 4.49(1 \mathrm{H}, \mathrm{d}, \mathrm{J}=9.5 \mathrm{~Hz}), 1.31$ (9H, s); ${ }^{13} \mathrm{C} \mathrm{NMR}\left(\mathrm{CDCl}_{3}\right): \square$ 170.36, 137.95, 137.32, 128.84, 128.79, 128.76, 128.39, 128.17, 127.68, 82.15, 80.61, 71.49, 28.34. Anal. Calc. for $\mathrm{C}_{19} \mathrm{H}_{22} \mathrm{O}_{3}$ : C, 76.48; H, 7.43; Found: C, 76.67; H, 7.62.

Benzyloxy-(4-nitro-phenyl)-acetic acid tert-butyl ester (table 4, entry 2) : ${ }^{1} \mathrm{H}$ NMR $\left(\mathrm{CDCl}_{3}\right): \square 8.13(2 \mathrm{H}, \mathrm{d}, \mathrm{J}=7.0 \mathrm{~Hz}), 7.57(2 \mathrm{H}, \mathrm{d}, \mathrm{J}=7.0 \mathrm{~Hz}), 7.23-7.28(5 \mathrm{H}, \mathrm{m}), 4.83$ $(1 \mathrm{H}, \mathrm{s}), 4.62(1 \mathrm{H}, \mathrm{d}, \mathrm{J}=9.4 \mathrm{~Hz}), 4.51(1 \mathrm{H}, \mathrm{d}, \mathrm{J}=9.4 \mathrm{~Hz}), 1.32(9 \mathrm{H}, \mathrm{s}) ;{ }^{13} \mathrm{C} \mathrm{NMR}$ $\left(\mathrm{CDCl}_{3}\right): \square$ 169.14, 148.31, 144.52, 137.23, 128.96, 128.55, 128.43, 128.26, 124.03, 83.17, 79.76, 72.18, 28.30. Anal. Calc. for $\mathrm{C}_{19} \mathrm{H}_{21} \mathrm{NO}_{5}$ : C, 66.46; H, 6.16; N, 4.08; Found: C, 66.54; H, 6.43; N, 4.01 .

Benzyloxy-(4-methoxy-phenyl)-acetic acid tert-butyl ester (table 4, entry 3): ${ }^{1} \mathrm{H}$ NMR $\left(\mathrm{CDCl}_{3}\right)$ : $\square 7.30-7.40(7 \mathrm{H}, \mathrm{m}), 6.90(2 \mathrm{H}, \mathrm{d}, \mathrm{J}=8.6 \mathrm{~Hz}), 4.77(1 \mathrm{H}, \mathrm{s}), 4.61(1 \mathrm{H}, \mathrm{d}, \mathrm{J}$ $=12.0 \mathrm{~Hz}), 4.58(1 \mathrm{H}, \mathrm{d}, \mathrm{J}=12.0 \mathrm{~Hz}), 3.83(3 \mathrm{H}, \mathrm{s}), 1.42(9 \mathrm{H}, \mathrm{s}) ;{ }^{13} \mathrm{C} \mathrm{NMR}\left(\mathrm{CDCl}_{3}\right): \square$ 170.62, 160.07, 138.00, 129.42, 129.06, 128.79, 128.41, 128.16, 114.26, 82.04, 80.04, 71.24, 55.67, 28.36. Anal. Calc. for $\mathrm{C}_{20} \mathrm{H}_{24} \mathrm{O}_{4}$ : C, 73.15; H, 7.37; Found: C, 73.33; H, 7.28 .

2-Benzyloxy-2-phenyl-propionic acid methyl ester (table 4, entry 4): ${ }^{1} \mathrm{H}$ NMR $\left(\mathrm{CDCl}_{3}\right): \square 7.56-7.59(2 \mathrm{H}, \mathrm{m}), 7.30-7.46(8 \mathrm{H}, \mathrm{m}), 4.59(1 \mathrm{H}, \mathrm{d}, \mathrm{J}=11.2 \mathrm{~Hz}), 4.45(1 \mathrm{H}, \mathrm{d}$, $\mathrm{J}=11.2 \mathrm{~Hz}), 3.77(3 \mathrm{H}, \mathrm{s}), 1.91(3 \mathrm{H}, \mathrm{s}) ;{ }^{13} \mathrm{C} \mathrm{NMR}\left(\mathrm{CDCl}_{3}\right): \square$ 174.07, 141.53, 138.89, $128.82,128.75,128.42,127.90,126.27,82.41,67.22,52.94,23.94$. Anal. Calc. for $\mathrm{C}_{17} \mathrm{H}_{18} \mathrm{O}_{3}$ : C, 75.53; H, 6.71; Found: C, 75.67; H, 6.79. 
(4-tert-Butyl-phenyl)-methoxy-acetic acid ethyl ester (table 4, entry 5): ${ }^{1} \mathrm{H}$ NMR $\left(\mathrm{CDCl}_{3}\right): \square 7.37-7.42(4 \mathrm{H}, \mathrm{m}), 4.75(1 \mathrm{H}, \mathrm{s}), 4.15-4.28(2 \mathrm{H}, \mathrm{m}), 3.42(3 \mathrm{H}, \mathrm{s}), 1.33(9 \mathrm{H}, \mathrm{s})$, $1.24(3 \mathrm{H}, \mathrm{t}, \mathrm{J}=7.1 \mathrm{~Hz}) ;{ }^{13} \mathrm{C} \mathrm{NMR}\left(\mathrm{CDCl}_{3}\right): \square 171.30,152.07,133.63,127.32,125.97$, 82.86, 61.61, 52.72, 35.01, 31.70, 14.55. Anal. Calc. for $\mathrm{C}_{15} \mathrm{H}_{22} \mathrm{O}_{3}$ : C, 71.97; $\mathrm{H}, 8.86$; Found: C, 72.41; H, 8.85.

(4-Methoxycarbonylphenyl)-methoxy-acetic acid ethyl ester (table 4, entry 6): ${ }^{1} \mathrm{H}$ NMR $\left(\mathrm{CDCl}_{3}\right)$ : $\square 8.06(2 \mathrm{H}, \mathrm{d}, \mathrm{J}=8.4 \mathrm{~Hz}), 7.55(2 \mathrm{H}, \mathrm{d}, \mathrm{J}=8.2 \mathrm{hz}), 4.83(1 \mathrm{H}, \mathrm{s}), 4.16-$ $4.25(2 \mathrm{H}, \mathrm{m}), 3.94(3 \mathrm{H}, \mathrm{s}), 3.46(3 \mathrm{H}, \mathrm{s}), 1.24(3 \mathrm{H}, \mathrm{t}, \mathrm{J}=7.1 \mathrm{~Hz}) ;{ }^{13} \mathrm{C} \mathrm{NMR}\left(\mathrm{CDCl}_{3}\right): \square$ $170.50,167.10,141.61,130.81,130.28,127.45,82.58,61.93,57.98,52.60,14.47$. Anal. Calc. for $\mathrm{C}_{13} \mathrm{H}_{16} \mathrm{O}_{5}$ : C, 61.90; H, 6.39; Found: C, 61.85; H, 6.40.

Arylation of the Trimethylsilyl ketenimines of the Evans Auxiliary. (4S, 2'S)-4isopropyl-3-(2'-phenyl-propanoyl)oxazolidin-2-one (table 3 , entries 1,2$):^{6}$ To a screw-capped vial containing $\mathrm{P}^{t} \mathrm{Bu}_{3}(0.500 \mathrm{M}$ solution in toluene, $200 \square \mathrm{L}, 0.010 \mathrm{mmol})$, $\mathrm{Pd}(\mathrm{dba})_{2}(29 \mathrm{mg}, 0.050 \mathrm{mmol}), \mathrm{ZnF}_{2}(52 \mathrm{mg}, 0.50 \mathrm{mmol})$ and phenyl bromide (157 mg, $1.00 \mathrm{mmol}$ ) was added the trimethylsilyl enolate 2 of the Evans imide $(370.0 \mathrm{mg}, 1.44$ mmol), followed by DMF (10 mL). The vial was sealed with a cap containing a PTFE septum and removed from the dry box. The heterogeneous reaction mixture was stirred at $80{ }^{\circ} \mathrm{C}$ for $12 \mathrm{~h}$. The crude reaction was then allowed to cool to room temperature and was diluted with $\mathrm{Et}_{2} \mathrm{O}$. The resulting solution was washed with $\mathrm{H}_{2} \mathrm{O}$. The organic phase was dried over $\mathrm{Na}_{2} \mathrm{SO}_{4}$, filtered, and concentrated at reduced pressure. Purification of the crude material by flash chromatography, eluting with 2\% EtOAc in hexanes, gave 67\% yield of the $\square$-aryl imide. ${ }^{1} \mathrm{H}$ NMR $\left(\mathrm{CDCl}_{3}\right)$ : $\square 7.27(2 \mathrm{H}, \mathrm{d}, J=7.4 \mathrm{~Hz}), 7.22(2 \mathrm{H}, \mathrm{t}, J=$ $7.3 \mathrm{~Hz}), 7.15(1 \mathrm{H}, \mathrm{t}, J=7.3 \mathrm{~Hz}), 5.06(1 \mathrm{H}, \mathrm{q}, J=7.0 \mathrm{~Hz}), 4.25-4.28(1 \mathrm{H}, \mathrm{m}), 4.00-4.07$ $(2 \mathrm{H}, \mathrm{m}), 2.33-2.37(1 \mathrm{H}, \mathrm{m}), 1.43(3 \mathrm{H}, \mathrm{d}, J=7.0 \mathrm{~Hz}), 0.84(3 \mathrm{H}, \mathrm{d}, J=6.6 \mathrm{~Hz}), 0.82(3 \mathrm{H}$, $\mathrm{d}, J=6.1 \mathrm{~Hz}) .{ }^{13} \mathrm{C} \mathrm{NMR}\left(\mathrm{CDCl}_{3}\right): \square$ 175.04, 153.99, 140.72, 128.98, 128.56, 127.59, $63.50,59.43,43.45,28.94,20.09,18.42,15.11$. 
4-tert-Butyl-3-(2-phenyl-propionyl)-oxazolidin-2-one (table 3, entries 3 and 4): ${ }^{1} \mathrm{H}$ $\operatorname{NMR}\left(\mathrm{CDCl}_{3}\right): \square 7.27-7.38(5 \mathrm{H}, \mathrm{m}), 5.18(1 \mathrm{H}, \mathrm{q}, J=7.0 \mathrm{~Hz}), 4.41(1 \mathrm{H}, \mathrm{dd}, \mathrm{J}=7.5$ and $1.3 \mathrm{~Hz}), 4.24(1 \mathrm{H}, \mathrm{dd}, \mathrm{J}=9.3$ and $1.4 \mathrm{~Hz}), 4.09(1 \mathrm{H}, \mathrm{dd}, \mathrm{J}=9.2$ and $7.6 \mathrm{~Hz}), 1.58(3 \mathrm{H}, \mathrm{d}$, $J=7.0 \mathrm{~Hz}), 0.98(9 \mathrm{H}, \mathrm{s}) .{ }^{13} \mathrm{C} \mathrm{NMR}\left(\mathrm{CDCl}_{3}\right): \square 175.16,154.65,140.53,128.96,128.60$, $127.60,65.41,61.78,43.38,36.22,26.15,20.71$.

3-[2-(3-Acetyl-phenyl)-propionyl]-4-isopropyl-oxazolidin-2-one (table 3, entry 5): ${ }^{1} \mathrm{H}$ NMR $\left(\mathrm{CDCl}_{3}\right.$, mixture of two isomers) $\square 7.94-7.95(1 \mathrm{H}, \mathrm{m}), 7.85-7.87(1 \mathrm{H}, \mathrm{m})$, 7.57-7.60 $(1 \mathrm{H}, \mathrm{m}), 7.43(1 \mathrm{H}, \mathrm{t}, \mathrm{J}=7.7 \mathrm{~Hz}), 5.15-5.24(1 \mathrm{H}, \mathrm{m}), 4.51(0.13 \mathrm{H}, \mathrm{dt}, \mathrm{J}=8.6$ and $3.5 \mathrm{~Hz})$, $4.40(0.87 \mathrm{H}, \mathrm{dt}, \mathrm{J}=7.9$ and $4.0 \mathrm{~Hz}), 4.12-4.30(2 \mathrm{H}, \mathrm{m}), 2.62(2.6 \mathrm{H}, \mathrm{s}), 2.61(0.4 \mathrm{H}, \mathrm{s})$, 2.40-2.56 (0.87H, m), 2.21-2.26 (0.13H, m), $1.56(2.6 \mathrm{H}, \mathrm{d}, \mathrm{J}=7.0 \mathrm{~Hz}), 1.51(0.4 \mathrm{H}, \mathrm{d}, \mathrm{J}=$ $7.0 \mathrm{~Hz}), 0.94(2.6 \mathrm{H}, \mathrm{d}, \mathrm{J}=7.0 \mathrm{~Hz}), 0.93(2.6 \mathrm{H}, \mathrm{d}, \mathrm{J}=6.9 \mathrm{~Hz}), 0.83(0.4 \mathrm{H}, \mathrm{d}, \mathrm{J}=7.1 \mathrm{~Hz})$, $0.49(0.4 \mathrm{H}, \mathrm{d}, \mathrm{J}=6.9 \mathrm{~Hz}) ;{ }^{13} \mathrm{C} \mathrm{NMR}\left(\mathrm{CDCl}_{3}\right.$, mixture of two isomers, major isomer reported): $\square$ 198.48, 174.57, 153.99, 141.19, 137.86, 133.54, 129.25, 128.49, 127.64, 63.59, 59.34, 43.48, 28.86, 27.16, 20.10, 18.41, 15.09. Anal. Calc. for $\mathrm{C}_{17} \mathrm{H}_{21} \mathrm{NO}_{4}$ : C, 67.31; H, 6.98; N, 4.62;; Found: C, 67.29; H, 7.24; N, 4.51.

4-Isopropyl-3-(2-o-tolyl-propionyl)-oxazolidin-2-one (table 3, entry 6): ${ }^{1} \mathrm{H}$ NMR $\left(\mathrm{CDCl}_{3}\right): \square 7.13-7.18(4 \mathrm{H}, \mathrm{m}), 5.19(1 \mathrm{H}, \mathrm{q}, J=6.9 \mathrm{~Hz}), 4.42-4.45(1 \mathrm{H}, \mathrm{m}), 4.12-4.19$ $(2 \mathrm{H}, \mathrm{m}), 2.46-2.51(1 \mathrm{H}, \mathrm{m}), 2.43(3 \mathrm{H}, \mathrm{s}), 1.46(3 \mathrm{H}, \mathrm{d}, J=7.0 \mathrm{~Hz}), 0.96(3 \mathrm{H}, \mathrm{d}, J=7.1$ $\mathrm{Hz}), 0.93(3 \mathrm{H}, \mathrm{d}, J=6.9 \mathrm{~Hz}) .{ }^{13} \mathrm{C} \mathrm{NMR}\left(\mathrm{CDCl}_{3}\right): \square 175.27,153.89,139.48,136.88$, 131.10, 127.38, 126.66, 125.90, 63.56, 59.57, 41.03, 28.97, 19.71, 18.71, 18.48, 15.11. Anal. Calc. for $\mathrm{C}_{16} \mathrm{H}_{21} \mathrm{NO}_{3}$ : C, 69.79; H, 7.69; N, 5.09; Found: C, 69.73; H, 7.72; N, 5.05.

\section{Experimental Procedure for the evaluation of epimerization during the $\square$-arylation:}

To a screw-capped vial containing $\mathrm{P}^{t} \mathrm{Bu}_{3}(0.500 \mathrm{M}$ solution in toluene, $20 \square \mathrm{L}, 0.010$ $\mathrm{mmol}), \mathrm{Pd}(\mathrm{dba})_{2}(2.9 \mathrm{mg}, 0.0050 \mathrm{mmol}), \mathrm{ZnF}_{2}(5.2 \mathrm{mg}, 0.050 \mathrm{mmol})$ and phenyl bromide (15.7 $\mathrm{mg}, 0.100 \mathrm{mmol}$ ) was added the trimethylsilyl enolate 2 of the Evans imide (37.0 $\mathrm{mg}, 0.144 \mathrm{mmol})$ and compound 1 (>90\% de), ${ }^{6}$ followed by DMF $(1.0 \mathrm{~mL})$. The vial was sealed with a cap containing a PTFE septum and removed from the dry box. The heterogeneous reaction mixture was stirred at $80{ }^{\circ} \mathrm{C}$ for $12 \mathrm{~h}$. The crude reaction was 
then allowed to cool to room temperature and was diluted with $\mathrm{Et}_{2} \mathrm{O}(5.0 \mathrm{~mL})$. The resulting solution was washed with $\mathrm{H}_{2} \mathrm{O}(5 \times 2.0 \mathrm{~mL})$. The organic phase was dried over $\mathrm{Na}_{2} \mathrm{SO}_{4}$, filtered, and concentrated at reduced pressure. The residue was then analyzed by ${ }^{1}$ H NMR spectroscopy. Only one set of signals was observed for compound $\mathbf{1}$ and its (2')epimer. ${ }^{6}$ From integration of the ${ }^{1} \mathrm{H}$ NMR signals, the diastereomeric ratio of the isopropyl derivative formed from the $\square$-arylation was $88: 12$. The same reaction on a 1.00 mmol scale in the absence of the added $\square$-aryl imide provided the major diastereomer in $67 \%$ yield after purification by flash chromatography eluting with $2 \%$ EtOAc in hexanes.

3-[2-(4-tert-Butyl-phenyl)-propionyl]-4-isopropyl-oxazolidin-2-one (table 3, entry 7): ${ }^{1} \mathrm{H}$ NMR $\left(\mathrm{CDCl}_{3}\right): \square 7.28-7.35$ (4H, m), $5.15(1 \mathrm{H}, \mathrm{q}, J=7.0 \mathrm{~Hz}), 4.36-4.40(1 \mathrm{H}, \mathrm{m})$, 4.13-4.18 (2H, m), $2.45(1 \mathrm{H}, \mathrm{m}), 1.53(3 \mathrm{H}, \mathrm{d}, J=7.0 \mathrm{~Hz}), 1.32(9 \mathrm{H}, \mathrm{s}), 0.94(3 \mathrm{H}, \mathrm{d}, J=$ $7.0 \mathrm{~Hz}), 0.93(3 \mathrm{H}, \mathrm{d}, J=7.0 \mathrm{~Hz}) .{ }^{13} \mathrm{C} \mathrm{NMR}\left(\mathrm{CDCl}_{3}\right): \square 175.29,154.04,150.37,137.54$, $128.16,125.89,63.46,59.40,42.84,34.85,31.76,28.91,20.09,18.45,15.11$. Anal. Calc. for $\mathrm{C}_{19} \mathrm{H}_{27} \mathrm{NO}_{3}$ : C, 71.89; H, 8.57; N, 4.41; Found: C, 72.28; H, 8.62; N, 4.39.

4-[2-(4-Isopropyl-2-oxo-oxazolidin-3-yl)-1-methyl-2-oxo-ethyl]-benzonitrile (table 3, entry 8, major isomer): ${ }^{1} \mathrm{H}$ NMR $\left(\mathrm{CDCl}_{3}\right)$ : $\square 7.61(2 \mathrm{H}, \mathrm{d}, \mathrm{J}=8.3 \mathrm{~Hz}), 7.47(2 \mathrm{H}, \mathrm{d}, \mathrm{J}=$ $8.4 \mathrm{~Hz}), 5.20(1 \mathrm{H}, \mathrm{q}, J=7.0 \mathrm{~Hz}), 4.38-4.41(1 \mathrm{H}, \mathrm{m}), 4.16-4.21(2 \mathrm{H}, \mathrm{m}), 2.39-2.47(1 \mathrm{H}$, m), $1.53(3 \mathrm{H}, \mathrm{d}, J=7.0 \mathrm{~Hz}), 0.94(3 \mathrm{H}, \mathrm{d}, J=7.0 \mathrm{~Hz}), 0.92(3 \mathrm{H}, \mathrm{d}, J=7.0 \mathrm{~Hz}) .{ }^{13} \mathrm{C} \mathrm{NMR}$ $\left(\mathrm{CDCl}_{3}\right)$ : $\square$ 173.83, 153.95, 145.88, 132.79, 129.52, 119.17, 111.54, 63.64, 59.32, 43.72, 28.80, 19.98, 18.38, 15.06. Anal. Calc. for $\mathrm{C}_{16} \mathrm{H}_{18} \mathrm{~N}_{2} \mathrm{O}_{3}$ : C, 67.12; H, 6.34; N, 9.78; Found: C, 66.86; H, 6.69; N, 9.61.

4-[2-(4-Isopropyl-2-oxo-oxazolidin-3-yl)-1-methyl-2-oxo-ethyl]-benzonitrile (table 3, entry 8, minor isomer): ${ }^{1} \mathrm{H}$ NMR $\left(\mathrm{CDCl}_{3}\right): \square 7.62(2 \mathrm{H}, \mathrm{d}, \mathrm{J}=8.3 \mathrm{~Hz}), 7.50(2 \mathrm{H}, \mathrm{d}, \mathrm{J}=$ $8.3 \mathrm{~Hz}), 5.18(1 \mathrm{H}, \mathrm{q}, J=6.9 \mathrm{~Hz}), 4.50(1 \mathrm{H}, \mathrm{dt}, \mathrm{J}=8.5$ and $3.5 \mathrm{~Hz}), 4.29(1 \mathrm{H}, \mathrm{t}, \mathrm{J}=9.0$ $\mathrm{Hz}), 4.16(1 \mathrm{H}, \mathrm{dd}, \mathrm{J}=9.2$ and $3.2 \mathrm{~Hz}), 2.18-2.24(1 \mathrm{H}, \mathrm{m}), 1.49(3 \mathrm{H}, \mathrm{d}, J=6.9 \mathrm{~Hz}), 0.84$ $(3 \mathrm{H}, \mathrm{d}, J=7.0 \mathrm{~Hz}), 0.52(3 \mathrm{H}, \mathrm{d}, J=6.9 \mathrm{~Hz}) .{ }^{13} \mathrm{C} \mathrm{NMR}\left(\mathrm{CDCl}_{3}\right): \square 173.58,153.88$, $146.20,132.82,129.37,119.12,111.55,63.57,58.65,43.89,28.42,19.08,18.19,14.61$. 
Anal. Calc. for $\mathrm{C}_{16} \mathrm{H}_{18} \mathrm{~N}_{2} \mathrm{O}_{3}: \mathrm{C}, 67.12 ; \mathrm{H}, 6.34 ; \mathrm{N}, 9.78$; Found: $\mathrm{C}, 66.80 ; \mathrm{H}, 5.98 ; \mathrm{N}$, 9.48 .

\section{Representative procedures for the arylation of the Ley auxiliary:}

5,6-Dimethoxy-5,6-dimethyl-3-phenyl-[1,4]dioxan-2-one (table 5, entry 1, 2): ${ }^{\mathbf{8}} 5,6$ -

Dimethoxy-5,6-dimethyl-[1,4]dioxan-2-one $\mathbf{8}^{8,9}(1.00 \mathrm{~g}, 5.26 \mathrm{mmol})$ was dissolved in anhydrous THF ( $20 \mathrm{~mL}$ ), and the mixture was cooled to $-78^{\circ} \mathrm{C}$ under $\mathrm{N}_{2}$. LDA $(3.2 \mathrm{~mL}$ of a $2.0 \mathrm{M}$ solution in THF/heptane, $6.4 \mathrm{mmol}$ ) was then added dropwise. After addition, the mixture was stirred for another $5 \mathrm{~min}$ before addition of $\mathrm{TMSCl}$ ( $0.83 \mathrm{~mL}, 6.6$ mmol). The resulting solution was then allowed to warm to room temperature overnight. The THF was then removed under reduced pressure, and hexanes $(20 \mathrm{~mL})$ was added to the residue. The resulting suspension was filtered through Celite, and the hexanes were removed in vacuo to afford $1.40 \mathrm{~g}$ of the desired trimethylsilyl ketene acetal (judged to be $90-95 \%$ pure from NMR). ${ }^{1} \mathrm{H}$ NMR $\left(\mathrm{CDCl}_{3}\right)$ : $\square 5.55(1 \mathrm{H}, \mathrm{s}), 3.41(3 \mathrm{H}, \mathrm{s}), 3.26(3 \mathrm{H}, \mathrm{s})$, $1.49(3 \mathrm{H}, \mathrm{s}), 1.42(3 \mathrm{H}, \mathrm{s}), 0.25(9 \mathrm{H}, \mathrm{s})$. This material was directly employed in the arylation step. In a dry box, to a screw cap vial was added $\mathrm{PhBr}(78.5 \mathrm{mg}, 0.500 \mathrm{mmol})$, $\mathrm{ZnF}_{2}(26.0 \mathrm{mg}, 0.0250 \mathrm{mmol}), \mathrm{Pd}(\mathrm{dba})_{2}(15.0 \mathrm{mg}, 0.00250 \mathrm{mmol})$ and $\mathrm{P}\left({ }^{\mathrm{t}} \mathrm{Bu}\right)_{3}(100 \square \mathrm{L}$, $0.5 \mathrm{M}$ solution in toluene, $0.005 \mathrm{mmol}$ ). The trimethylsilyl ketene acetal prepared above ( $170 \mathrm{mg}$ calculated amount according to the purity, $0.650 \mathrm{mmol}$ ) was added to the mixture followed by $4.0 \mathrm{~mL}$ DMF. The vial was then sealed and heated at $80^{\circ} \mathrm{C}$ for $12 \mathrm{~h}$. The solution was then allowed to cool to room temperature and partitioned in ethyl ether $(50 \mathrm{~mL})$ and water $(10 \mathrm{~mL})$. The ether layer was washed with water and brine and dried over $\mathrm{Na}_{2} \mathrm{SO}_{4}$. After removing the ether, the residue was subjected to automated flash chromatography using $8 \%$ EtOAc/hexanes to provide the desired product $(88.4 \mathrm{mg})$ in $66 \%$ yield. ${ }^{1} \mathrm{H}$ NMR $\left(\mathrm{CDCl}_{3}\right): \square 7.59(2 \mathrm{H}, \mathrm{d}, \mathrm{J}=6.8 \mathrm{~Hz}), 7.32-7.41(3 \mathrm{H}, \mathrm{m}), 5.21(1 \mathrm{H}, \mathrm{s})$, $3.47(3 \mathrm{H}, \mathrm{s}), 3.45$ (3H, s), $1.58(3 \mathrm{H}, \mathrm{s}), 1.52(3 \mathrm{H}, \mathrm{s}) ;{ }^{13} \mathrm{C} \mathrm{NMR}\left(\mathrm{CDCl}_{3}\right): \mathrm{Q} 168.85$, 136.44, 129.01, 128.91, 128.22, 105.65, 98.98, 74.07, 50.46, 49.78, 18.40, 17.47.

\section{5,6-Dimethoxy-5,6-dimethyl-3-naphthalen-2-yl-[1,4]dioxan-2-one (table 5, entry 3):}

${ }^{1} \mathrm{H} \mathrm{NMR}\left(\mathrm{CDCl}_{3}\right)$ : $\square 8.07(1 \mathrm{H}, \mathrm{s}), 7.86-7.89(3 \mathrm{H}, \mathrm{m}), 7.72(1 \mathrm{H}, \mathrm{dd}, \mathrm{J}=8.6$ and $1.7 \mathrm{~Hz})$, 7.49-7.51 (2H, m), $5.39(1 \mathrm{H}, \mathrm{s}), 3.50(3 \mathrm{H}, \mathrm{s}), 3.49(3 \mathrm{H}, \mathrm{s}), 1.61(3 \mathrm{H}, \mathrm{s}), 1.57(3 \mathrm{H}, \mathrm{s}) ;{ }^{13} \mathrm{C}$ 
$\operatorname{NMR}\left(\mathrm{CDCl}_{3}\right)$ : $\square 168.83,133.81,133.78,133.55,128.76,128.65,128.07,127.80$,

126.74, 126.56, 125.52, 105.74, 99.10, 74.22, 50.50, 49.84, 18.44, 17.51. Anal. Calc. for $\mathrm{C}_{18} \mathrm{H}_{20} \mathrm{O}_{5}$ : C, 68.34; H, 6.37; Found: C, 68.08; H, 6.02 .

4-(5,6-Dimethoxy-5,6-dimethyl-3-oxo-[1,4]dioxan-2-yl)-benzoic acid methyl ester (table 5, entries 4, 5): ${ }^{1} \mathrm{H}$ NMR $\left(\mathrm{CDCl}_{3}\right)$ : $\square 8.06(2 \mathrm{H}, \mathrm{d}, \mathrm{J}=8.4 \mathrm{~Hz}), 7.70(2 \mathrm{H}, \mathrm{d}, \mathrm{J}=8.3$ $\mathrm{Hz}), 5.28(1 \mathrm{H}, \mathrm{s}), 3.93(3 \mathrm{H}, \mathrm{s}), 3.45(3 \mathrm{H}, \mathrm{s}), 3.43(3 \mathrm{H}, \mathrm{s}), 1.58(3 \mathrm{H}, \mathrm{s}), 1.54(3 \mathrm{H}, \mathrm{s}) ;{ }^{13} \mathrm{C}$ NMR ( $\left.\mathrm{CDCl}_{3}\right)$ : $\square 168.22,167.22,141.21,130.63,130.09,128.09,105.74,99.08,73.58$, 52.60, 50.50, 49.88, 18.36, 17.44. Anal. Calc. for $\mathrm{C}_{16} \mathrm{H}_{20} \mathrm{O}_{7}$ : C, 59.25; H, 6.22; Found: $\mathrm{C}$, $59.35 ; \mathrm{H}, 6.18$.

5,6-Dimethoxy-5,6-dimethyl-3-(3-nitro-phenyl)-[1,4]dioxan-2-one (table 5, entries 6, 7, 8): ${ }^{1} \mathrm{H}$ NMR $\left(\mathrm{CDCl}_{3}\right)$ : $\square 8.59(1 \mathrm{H}, \mathrm{t}, \mathrm{J}=1.9 \mathrm{~Hz}), 8.21(1 \mathrm{H}, \mathrm{ddd}, \mathrm{J}=8.2$ and 2.2 and 1.0 Hz), 7.92-7.94 (1H, m), $7.56(1 \mathrm{H}, \mathrm{t}, \mathrm{J}=8.0 \mathrm{~Hz}), 5.31(1 \mathrm{H}, \mathrm{s}), 3.47(3 \mathrm{H}, \mathrm{s}), 3.46(3 \mathrm{H}, \mathrm{s})$, $1.60(3 \mathrm{H}, \mathrm{s}), 1.56(3 \mathrm{H}, \mathrm{s}) ;{ }^{13} \mathrm{C} \mathrm{NMR}\left(\mathrm{CDCl}_{3}\right)$ : $\square 167.92,148.77,138.58,134.37,129.72$, $123.90,123.28,105.91,99.26,73.06,50.60,49.96,18.28,17.41$. Anal. Calc. for $\mathrm{C}_{14} \mathrm{H}_{17} \mathrm{NO}_{7}$ : C, 54.02; H, 5.50; N, 4.50; Found: C, 53.71; H, 5.31; N, 4.19.

3-(3-Acetyl-phenyl)-5,6-dimethoxy-5,6-dimethyl-[1,4]dioxan-2-one (table 5, entry 9): ${ }^{1} \mathrm{H} \mathrm{NMR}\left(\mathrm{CDCl}_{3}\right): \square 8.23(1 \mathrm{H}, \mathrm{t}, \mathrm{J}=1.7 \mathrm{~Hz}), 7.95(1 \mathrm{H}, \mathrm{dt}, \mathrm{J}=7.8$ and $1.3 \mathrm{~Hz}), 7.79(1 \mathrm{H}$, d, J = 7.7 Hz), $7.49(1 \mathrm{H}, \mathrm{t}, \mathrm{J}=7.7 \mathrm{~Hz}), 5.27(1 \mathrm{H}, \mathrm{s}), 3.49(3 \mathrm{H}, \mathrm{s}), 3.46(3 \mathrm{H}, \mathrm{s}), 2.63(3 \mathrm{H}$, s), $1.59(3 \mathrm{H}, \mathrm{s}), 1.54(3 \mathrm{H}, \mathrm{s}) ;{ }^{13} \mathrm{C} \mathrm{NMR}\left(\mathrm{CDCl}_{3}\right)$ : $\square 198.14,168.53,137.74,137.16$, 132.92, 129.23, 128.89, 128.36, 105.80, 99.10, 73.70, 50.59, 49.89, 27.11, 18.36, 17.46. Anal. Calc. for $\mathrm{C}_{16} \mathrm{H}_{20} \mathrm{O}_{6}$ : C, 62.33; H, 6.54; Found: C, 62.52; H, 6.70 .

5,6-Dimethoxy-3-(4-methoxy-phenyl)-5,6-dimethyl-[1,4]dioxan-2-one (table 5, entry 10): ${ }^{1} \mathrm{H}$ NMR ( $\left.\mathrm{CDCl}_{3}\right)$ : $\square 7.50(2 \mathrm{H}, \mathrm{d}, \mathrm{J}=8.7 \mathrm{~Hz}), 6.92(2 \mathrm{H}, \mathrm{d}, \mathrm{J}=8.8 \mathrm{~Hz}), 5.15(1 \mathrm{H}, \mathrm{s})$, $3.83(3 \mathrm{H}, \mathrm{s}), 3.48(3 \mathrm{H}, \mathrm{s}), 3.44(3 \mathrm{H}, \mathrm{s}), 1.57(3 \mathrm{H}, \mathrm{s}), 1.50(3 \mathrm{H}, \mathrm{s}) ;{ }^{13} \mathrm{C} \mathrm{NMR}\left(\mathrm{CDCl}_{3}\right):$ 169.15, 160.25, 129.49, 128.66, 114.38, 105.59, 98.96, 73.69, 55.71, 50.44, 49.75, 18.39, 17.48. Anal. Calc. for $\mathrm{C}_{15} \mathrm{H}_{20} \mathrm{O}_{6}: \mathrm{C}, 60.80 ; \mathrm{H}, 6.80$; Found: $\mathrm{C}, 60.82 ; \mathrm{H}, 6.71$. 
3-(2-Chloro-phenyl)-5,6-dimethoxy-5,6-dimethyl-[1,4]dioxan-2-one (table 5, entry 11): ${ }^{1} \mathrm{H}$ NMR $\left(\mathrm{CDCl}_{3}\right)$ : $7.58-7.60(1 \mathrm{H}, \mathrm{m}), 7.41-7.44(1 \mathrm{H}, \mathrm{m}), 7.28-7.32(2 \mathrm{H}, \mathrm{m}), 5.76$ $(1 \mathrm{H}, \mathrm{s}), 3.55(3 \mathrm{H}, \mathrm{s}), 3.48(3 \mathrm{H}, \mathrm{s}), 1.59(3 \mathrm{H}, \mathrm{s}), 1.48(3 \mathrm{H}, \mathrm{s}) ;{ }^{13} \mathrm{C} \mathrm{NMR}\left(\mathrm{CDCl}_{3}\right)$ : $168.10,134.82$, 134.68, 130.49, 130.23, 130.14, 127.74, 106.05, 99.04, 70.37, 50.59, 49.83, 18.25, 17.48. Anal. Calc. for $\mathrm{C}_{14} \mathrm{H}_{17} \mathrm{ClO}_{5}$ : C, 55.91; H, 5.70; Found: C, 55.90; H, 5.64.

5,6-Dimethoxy-5,6-dimethyl-2-naphthalen-2-yl-[1,4]dioxan-2-one (table 1, entry 12): ${ }^{1} \mathrm{H}$ NMR $\left(\mathrm{CDCl}_{3}\right): \square 8.31(1 \mathrm{H}, \mathrm{d}, \mathrm{J}=8.5 \mathrm{~Hz}), 7.88(2 \mathrm{H}, \mathrm{d}, \mathrm{J}=8.2 \mathrm{~Hz}), 7.75(1 \mathrm{H}, \mathrm{dd}, \mathrm{J}=$ 7.1 and $0.7 \mathrm{~Hz}), 7.48-7.60(3 \mathrm{H}, \mathrm{m}), 5.90(1 \mathrm{H}, \mathrm{s}), 3.60(3 \mathrm{H}, \mathrm{s}), 3.54(3 \mathrm{H}, \mathrm{s}), 1.64(3 \mathrm{H}, \mathrm{s})$, $1.54(3 \mathrm{H}, \mathrm{s}) ;{ }^{13} \mathrm{C} \mathrm{NMR}\left(\mathrm{CDCl}_{3}\right): \square 168.70,134.46,132.34,131.77,130.14,129.13$, $127.54,126.96,126.21,125.65,124.56,106.22$, 99.34, 71.42, 50.50, 49.90, 18.44, 17.58 . Anal. Calc. for $\mathrm{C}_{18} \mathrm{H}_{20} \mathrm{O}_{5}$ : C, 68.34; H, 6.37; Found: C, 68.63; H, 6.56.

\section{Procedure for conducting the reactions without a glovebox: Procedure for} conducting the reactions without a glovebox: To a round-bottom flask was added $\mathrm{Pd}(\mathrm{dba})_{2}(2.9 \mathrm{mg}, 0.0050 \mathrm{mmol}), \mathrm{Zn}\left(\mathrm{O}^{t} \mathrm{Bu}\right)_{2}(21.0 \mathrm{mg}$ of commercial material, which is about $50 \% \mathrm{H}_{2} \mathrm{O}$ by weight), the trimethylsilyl enolate of the Ley auxiliary (34.0 $\mathrm{mg}$ calculated according to the purity, $0.130 \mathrm{mmol})$ and 3-bromonitrobenzene $(20.2 \mathrm{mg}$, $0.100 \mathrm{mmol})$. The flask was purged with $\mathrm{N}_{2}$ for $5 \mathrm{~min}$ before addition of $\mathrm{P}\left({ }^{t} \mathrm{Bu}\right)_{3}(20 \mathrm{~mL}$ of $0.5 \mathrm{M}$ solution in toluene, $0.010 \mathrm{mmol})$. DMF $(1.0 \mathrm{~mL})$ was then added, and the resulting mixture was stirred for $12 \mathrm{~h}$. Trimethoxybenzene (12 $\mathrm{mg}, 0.071 \mathrm{mmol})$ as internal standard was then added to the solution, and the mixture was partitioned with $\mathrm{Et}_{2} \mathrm{O}(5.0 \mathrm{~mL})$ and $\mathrm{H}_{2} \mathrm{O}(2.0 \mathrm{~mL})$. The ether layer was washed with water, and the ether was evaporated in vacuo. The yield by NMR spectroscopy was determined with an internal standard to be within experimental error of quantitative, which is similar to that observed when the reactions are assembled in a drybox.

\section{References:}

(1) Hoffman, R.; Kim, H. O. J. Org. Chem. 1988, 53, 3855.

(2) Fuentes, L. M.; Shinkai, I.; Salzmann, T. N. J. Am. Chem. Soc. 1986, 108, 4675. 
(3) Jorgensen, M.; Lee, S.; Liu, X.; Wolkowski, J. P.; Hartwig, J. F. J. Am. Chem. Soc. 2002, 124, 12557.

(4) RajanBabu, T. V.; Chenard, B. L.; Petti, M. A. J. Org. Chem. 1986, 51, 1704.

(5) Jackson, W. R.; Rae, I. D.; Wong, M. G. Aust. J. Chem. 1986, 39, 303.

(6) Fukuzawa, S.; Chino, Y.; Yokoyama, T. Tetrahedron: Asymmetry 2002, 13, 1645.

(7) Bull, S. D.; Davies, S. G.; Key, M.; Nicholson, R. L.; Savory, E. D. Chem. Commun. 2000, 18, 1721.

(8) Ley, Steven V.; Michel, Patrick. Synlett 2001, 11, 1793.

(9) Diez, E.; Dixon, D. J.; Ley, S. V. Angew. Chem. Int. Ed. 2001, 40, 2906. 